\title{
IgG Clearance
}

National Cancer Institute

\section{Source}

National Cancer Institute. Ig G Clearance. NCI Thesaurus. Code C147373.

The determination of the clearance of endogenous IgG, used for evaluating the glomerular filtration rate. 